\title{
Circulating microRNAs as biomarkers for Sepsis secondary to pneumonia diagnosed via Sepsis 3.0
}

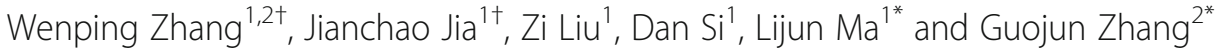

\begin{abstract}
Background: Sepsis biomarkers have limited specificity and sensitivity. Few studies have investigated microRNA (miRNA) biomarkers for sepsis secondary to pneumonia. This study aims to investigate the diagnostic and prognostic values of miRNAs in sepsis secondary to pneumonia.

Methods: Sepsis 3.0 was used to diagnose sepsis. Screening was performed through the Agilent miRNA chip technology by using the following criteria: $p<0.05$, fold $\geq 2$ or $<0.5$, or copy number $>50$ change. This study recruited 52 patients with pneumonia, including 31 males (59.6\%) and 21 females (40.4\%), 44 patients with sepsis secondary to pneumonia were diagnosed via Sepsis 3.0 (34 [77.3\%] males and 10 [22.7\%] females), and 21 healthy controls were used for miRNA verification. The miRNA levels were detected through fluorescence real-time quantitative polymerase chain reaction (qRT-PCR). Results: Fluorescence qRT-PCR detection showed that the miR7110-5p and miR-223-3p expression levels in both patient groups were upregulated compared with those in the healthy controls. The expression levels differed between patients with pneumonia and those with sepsis secondary to pneumonia. The sensitivity and specificity of miR-7110-5p to differentiate sepsis from healthy controls were 84.2 and $90.5 \%$, whereas those of miR-223-3p were 82.9 and $100 \%$, respectively. Multivariate analysis of variance suggested that the presence of sepsis affected the miR-223-3p level $(p=0.041)$, whereas the presence of sepsis $(p=$ $0.000)$ and the underlying disease $(p=0.025)$ influenced the miR-7110-5p level.
\end{abstract}

Conclusions: MiR-223-3p could be utilized to predict sepsis secondary to pneumonia.

Keywords: Sepsis, SOFA score, Pneumonia, miR-7110-5p, miR-223-3p, Sepsis 3.0

\section{Background}

Sepsis is a common complication that involves severe trauma, shock, infection, and surgery; it can lead to septic shock and multiple organ dysfunction syndrome (MODS). Sepsis is also a major cause of death in critical patients. It remains a remarkable challenge in clinical medicine because of its high mortality and morbidity rates. In developed countries, the incidence of sepsis reaches 100/ 100,000 [1], and approximately $2 \%$ of hospitalized patients

\footnotetext{
* Correspondence: malijun0401@163.com; 793392853@qq.com

${ }^{+}$Wenping Zhang and Jianchao Jia contributed equally to this work. Wenping

Zhang is the first author and Jianchao Jia should be considered co-first author.

'Department of Respiratory and Critical Care Medicine, The People's Hospital of Zhengzhou University, Zhengzhou, Henan, China

${ }^{2}$ Department of Respiratory and Critical Care Medicine, The First Affiliated Hospital of Zhengzhou University, No.1, East Jianshe Road, Zhengzhou 450052, Henan, China
}

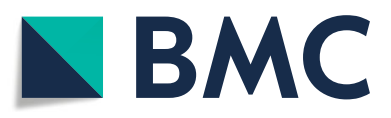

(c) The Author(s). 2019 Open Access This article is distributed under the terms of the Creative Commons Attribution 4.0 International License (http://creativecommons.org/licenses/by/4.0/), which permits unrestricted use, distribution, and

reproduction in any medium, provided you give appropriate credit to the original author(s) and the source, provide a link to the Creative Commons license, and indicate if changes were made. The Creative Commons Public Domain Dedication waiver (http://creativecommons.org/publicdomain/zero/1.0/) applies to the data made available in this article, unless otherwise stated.

are diagnosed during admission [2, 3]. With advancements in critical medicine, evidence-based medicine is widely used in clinical practices to address sepsis and its related conditions. However, approximately $1 / 5-1 / 2$ of the patient population with sepsis dies of MODS $[4,5]$.

MicroRNAs (miRNAs) are endogenous noncoding small RNAs of approximately 21-25 nucleotides in length. In 1993, Lee [6] discovered the first miRNA (lin-4) miRNAs in new Caenorhabditis species. Since then, thousands of miRNAs have been found. miRNAs can inhibit post-transcriptional gene expression or promote targeted mRNA degradation but cannot encode a protein. miRNAs can be detected in different fluids, such as blood, sweat, and urine. Whole panels of deregulated miRNAs have been described in the blood of patients with inflammatory/infectious diseases, thereby suggesting that circulating miRNAs might be suitable biomarkers in sepsis [7] 
but not in patients with respiratory infection. This study aimed to identify the differences in microRNA expression in patients affected with pneumonia and with sepsis secondary to pneumonia.

\section{Methods}

\section{Subject}

We conducted a prospective observational study to identify the differences in microRNA expression in patients affected with pneumonia and with sepsis secondary to pneumonia. An Agilent miRNA chip was used to screen the plasma of five patients with pneumonia and five patients with sepsis secondary to pneumonia. Fluorescence quantitative reverse-transcription polymerase chain reaction (qRT-PCR) was applied to test the differences in miRNA levels among the patients with pneumonia, those with sepsis secondary to pneumonia, and the control group, which were included from August 2016 to January 2017. Pneumonia was diagnosed in accordance with the diagnosis and treatment guidelines of hospital-acquired pneumonia (HAP) and community-acquired pneumonia (CAP) drafted by the American Thoracic Society and the Infectious Diseases Society of America in 2007 and 2016 $[8,9]$. Sepsis secondary to pneumonia was diagnosed based on the Sepsis 3.0 guideline, which can be simplified as sepsis $=$ infection + sequential organ failure assessment $($ SOFA) $\geq 2$ [10]. We used an adjudication procedure according to the guideline. For each case, three respiratory specialists independently conducted the diagnosis, and the cases with consistent diagnosis were included in the analysis. The Ethics Committee approved this study. All patients or guardians signed an informed consent form. Patients were excluded according to the following criteria: 1) age $<18$ years; 2) death within $24 \mathrm{~h}$ of admission; 3 ) neutrophil count $\leq 0.5 \times 10^{9} / \mathrm{L}$; 4) HIV/AIDS; and 5) unwillingness to participate in this study.

\section{miRNA gene chip analysis}

Agilent Human miRNA chip V21.0 provided data from the miRNA database (miRBase) version 21.0 and covered 2661 human-related miRNAs. We conducted statistical analysis control to avoid false discovery caused by individual bias and experimental factors. The coefficient of variance $(\mathrm{CV})$ for the repeated probe (10 repetitions) signal was not more than $7 \%$ with an acceptance value of $<15 \%$. Horizontal evaluation of the chip quality suggested that the chip was stable. Cluster analysis and biology repeat sample correlation analysis showed that the samples in the pneumonia group and the other group exhibited good correlation.

qRT-PCR analysis was conducted to detect the expression levels of the selected miRNAs in healthy controls, patients with pneumonia, and patients with sepsis secondary to pneumonia. RNA was extracted from the plasma by using an miRcute serum/plasma miRNA isolation kit (TianGen, Biotech Company, Beijing, China), which relatively enriched the small RNAs. qRT-PCR analysis was also performed using SYBR Green I fluorescent dye in a miScript $\mathrm{SYBR}^{\circ}$ Green PCR kit (Qiagen, Valencia, CA). All procedures were conducted in accordance with the manufacturer's instructions and were repeated thrice. The reverse primer in the miRcute miRNA qPCR detection kit (SYBR) was similar for all miRNAs, but the forward primers used were different. The forward primers for the miRNAs were synthesized by the Sangon Biotech Company. An ABI PRISM 7300 detection system was utilized for qRT-PCR analysis. The miRNA sequence is listed in Table 1.

\section{Statistical analysis}

Data were statistically analyzed using SPSS 22.0. In the univariate analysis, data with normal and non-normal distributions and the count data were evaluated through t-test, Mann-Whitney test, and $x^{2}$ test, respectively. $p<$ 0.05 indicated significant differences. The specificity and sensitivity of miRNAs in the diagnosis of sepsis were calculated in terms of the area under the receiver operating characteristic (ROC) curve.

\section{Results}

\section{miRNA gene chip analysis}

Six miRNAs, namely, miR-4800-5p, miR-6510-5p, miR-6740-5p, miR-7110-5p, miR-765, and miR-940, satisfied the screening criteria of $p<0.01$, fold change $\geq 2$ or $<0.5$, and copy number $>50$. Among these miRNAs, miR-940 expression was downregulated in the sepsis group (Fig. 1). Two miRNAs, namely, miR-6740-5p and miR-7110-5p, also met the screening criteria and were upregulated in the sepsis group.

\section{Clinical characteristics of the subjects}

Exactly 52 patients with pneumonia, including 31 males (59.6\%) and 21 females (40.4\%) with an average age of $52.10 \pm 16.15$ years old, were enrolled in this study. Fifty (96.1\%) patients were diagnosed with CAP, and two patients (3.8\%) were diagnosed with HAP. Exactly 44 patients with sepsis secondary to pneumonia, including 34 males (77.3\%) and 10 females $(22.7 \%)$ with an average age of $63.72 \pm$ 19.83 years old, were diagnosed by applying the Sepsis 3.0 criteria. Exactly 29 patients (65.9\%) were diagnosed with CAP, and 15 patients (34.1\%) were diagnosed with HAP/ VAP (ventilator-associated pneumonia). Among the 44 sepsis cases, $42(95.5 \%)$ had a definite etiology. They were due to bacteria (23,52.3\%), viruses (4, 9.1\%), Aspergillus (2, $4.5 \%)$, and multiple pathogens (13, 29.5\%). Among the sepsis cases with multiple pathogens, seven cases were attributed to bacteria with fungi. Three cases were attributed to more than two different bacterial strains. Two cases were 
Table 1 miRNAs and Primer sequence

\begin{tabular}{lll}
\hline miRNAs & miRNAs sequences & Primer \\
\hline hsa-miR-4800-5p & AGUGGACCGAGGAGGAAGGA & AGTGGACCGAGGAAGGAAGGA \\
hsa-miR-6510-5p & CAGCAGGGGAGAGAGAGGAGUC & CAGCAGGGGAGAGAGAGGAGTC \\
hsa-miR-6740-5p & AGUUUGGGAUGGAGAGAGGAGA & AGTTGGGATGGAGAGAGGAGA \\
hsa-miR-7110-5p & UGGGGGUUGGGGAGAGAGG & TGGGGGGTGGGGAGAGAGAG \\
hsa-miR-765 & UGGAGGAGAAGGAAGGUGAUG & TGGAGGAGAAGGAAGGTGATG \\
hsa-miR-940 & AAGGCAGGGCCCCCGCUCCCC & AAGGCAGGGCCCCCGCTCCCC \\
hsa-miR-150-5p & UCUCCCAACCCUUGUACCAGUG & TCTCCCAACCCTTGACCAGTG \\
hsa-miR-223-3p & UGUCAGUUUGUCAAAUACCCCA & UGUCAGUUUGUCAAAUACCCCA \\
hsa-miR-122-5p & UGGAGUGUGACAAUGGUGUUUG & TGGAGTGTGACAATGGTGTTG \\
hsa-miR-25-3p & CAUUGCACUUGUCUCGGUCUGA & CATTGCACTGTCTCGGTCTGA \\
hsa-miR-16-5p & UAGCAGCACGUAAAUAUUGGCG & TAGCAGCACGTAAATATTGGCG \\
miR universal primer $R$ & & GATCCAGTCTCAGGGTCCGAG \\
\hline
\end{tabular}

attributed to virus with fungi, and one case to bacteria with virus. The SOFA score of patients with sepsis secondary to pneumonia was $6.47 \pm 3.11$. The clinical characteristics of all patients are presented in Table 2 . Exactly 21 healthy controls were volunteer doctors and nurses in our hospital.

\section{RT-PCR validation of miRNAs}

The results of the gene chip analysis indicated the presence of miR-4800-5p, miR-6510-5p, miR-6740-5p, miR-7110-5p, miR-765, and miR-940, which were rarely mentioned in previously published data. In this regard, relative data regarding miRNAs in sepsis were reviewed. miR-150, miR-223-3p, miR-25, and miR-122, which have been intensively studied but remained controversial in terms of their relationship with sepsis, were selected for RT-PCR validation. Ten miRNAs, including miR-4800-5p, miR-6510-5p, miR-6740-5p, miR-7110-5p, miR-765, and miR-940 satisfied the screening criteria and were selected as primary genes for the tests. The four remaining miRNAs, namely, miR-150, miR-223-3p, miR-25, and miR-122 were related to sepsis. miR-16 was used as the reference gene. Table 3 shows the $\Delta C T$ values of the 10 miRNAs in the healthy controls, patients with pneumonia, and patients with sepsis secondary to pneumonia. qRT-PCR results indicated that the expression levels of miR-7110-5p and miR-223-3p differed between the two patient groups and were upregulated in the plasma of patients with sepsis secondary to pneumonia. miR-7110-5p and miR-223-3p expression levels were higher in patients with pneumonia and sepsis than those in healthy controls (Figs. 2 and 3).

Considering the differences in the age and presence of comorbidities in pneumonia versus sepsis due to pneumonia, multivariate analysis of variance (MANOVA) was conducted to affirm whether the differences in miRNA expression were due to the presence of sepsis. In MANOVA, the variable factors include age $\geq 60$ years or not, merged underlying disease or not, and sepsis or not. The presence of sepsis affected the level of miR-223-3P $(p=0.041)$, whereas the presence of sepsis $(p=0.000)$ and underlying disease $(p=0.025)$ affected the level of miR-7110-5p. Further analysis cannot be conducted, because the variety of underlying diseases and number of cases are limited.

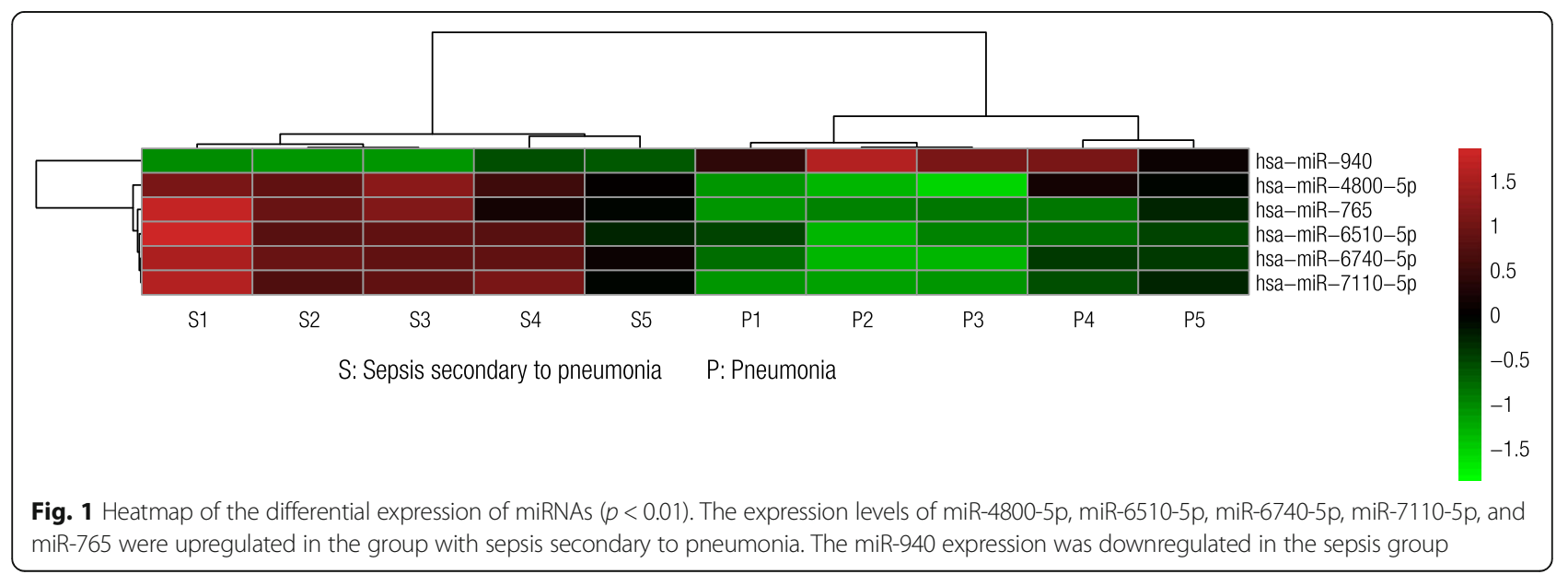


Table 2 Clinical characteristic of patients with pneumonia and sepsis secondary to pneumonia

\begin{tabular}{|c|c|c|c|c|}
\hline & & Pneumonia $(n)$ & Sepsis secondary to pneumonia $(n)$ & $P$ value \\
\hline \multirow[t]{2}{*}{ Sex } & Male & 31 & 34 & 0.065 \\
\hline & Female & 21 & 10 & \\
\hline \multicolumn{2}{|l|}{ Age } & $52.10 \pm 16.15$ & $63.72 \pm 19.83$ & 0.002 \\
\hline \multicolumn{2}{|c|}{ Merges more than 1 kind of underlying disease } & $6(11.50 \%)$ & $34(77.30 \%)$ & 0.000 \\
\hline \multicolumn{2}{|l|}{ SOFA score } & & $6.47 \pm 3.11$ & \\
\hline \multicolumn{2}{|l|}{ SOFA score above baseline } & & $5.84 \pm 2.92$ & \\
\hline \multirow[t]{2}{*}{ Prognosis } & Survivor & $52(100 \%)$ & $23(52.30 \%)$ & \\
\hline & Dead & $0(0 \%)$ & $21(47.70 \%)$ & \\
\hline \multirow[t]{6}{*}{ Major underlying comorbidities } & Diabetes & 0 & $9(20.45 \%)$ & \\
\hline & Acute cerebral infarction or cerebral hemorrhage & $2(3.85 \%)$ & $9(20.45 \%)$ & \\
\hline & chronic renal insufficiency & $3(3.85 \%)$ & $5(11.36 \%)$ & \\
\hline & coronary heart disease & 0 & $5(11.36 \%)$ & \\
\hline & autoimmune disease & $1(1.92 \%)$ & $3(6.82 \%)$ & \\
\hline & Chest trauma or surgery & 0 & $3(6.82 \%)$ & \\
\hline
\end{tabular}

\section{Identification of diagnostic and prognostic predictors for sepsis}

The area under the curve (AUC) of the ROC of miR-7110-5p for the prediction of pneumonia was 0.687 (Fig. 4). At a cutoff value of 3.66, miR-7110-5p yielded a sensitivity of $52.3 \%$ and a specificity of $100 \%$. The AUC of the ROC of miR-7110-5p for the prediction of sepsis secondary to pneumonia was 0.883 (Fig. 4). At a cutoff value of 4.41 , miR-7110-5p yielded a sensitivity of $84.2 \%$ and a specificity of $90.5 \%$. The AUC of the miR-223-3p ROC for the prediction of pneumonia was 0.909 (Fig. 5). At a cutoff value of 3.246, miR-223-3p yielded a sensitivity of $78.7 \%$ and a specificity of $94.7 \%$. The AUC of the miR-223-3p ROC for the prediction of sepsis secondary to pneumonia was 0.964 (Fig. 5). At a cutoff value of 2.759 , miR-223-3p yielded a sensitivity of $82.9 \%$ and a specificity of $100 \%$.

Table 3 RT-PCR of the 10 miRNAs in patients with pneumonia and sepsis secondary to pneumonia

\begin{tabular}{llll}
\hline & $\Delta C T$ & $P$ value \\
& Pneumonia & Sepsis secondary to pneumonia & \\
\hline miR-223-3p & $2.39 \pm 1.36$ & $1.44 \pm 1.43$ & 0.002 \\
miR-7110-5p & $4.15 \pm 2.52$ & $2.27 \pm 2.64$ & 0.001 \\
miR-940 & $3.19 \pm 1.84$ & $2.92 \pm 1.96$ & 0.493 \\
miR-4800-5p & $6.08 \pm 2.81$ & $6.11 \pm 2.54$ & 0.967 \\
miR-150 & $4.29 \pm 2.11$ & $4.66 \pm 1.90$ & 0.406 \\
miR-25 & $-2.28 \pm 1.86$ & $-2.33 \pm 2.10$ & 0.914 \\
miR-122 & $2.70 \pm 2.30$ & $2.20 \pm 2.06$ & 0.332 \\
miR-6740-5p & $5.15 \pm 3.00$ & $6.03 \pm 3.15$ & 0.231 \\
miR-765 & $5.06 \pm 3.12$ & $5.24 \pm 2.97$ & 0.822 \\
miR-6510-5p & $3.62 \pm 1.74$ & $3.77 \pm 1.83$ & 0.756 \\
\hline
\end{tabular}

The SOFA score could indicate the severity of sepsis. The $\Delta C T$ of miR-223-3p was $1.763 \pm 1.401$ at SOFA scores $\leq 6$ and was $0.762 \pm 1.308$ at SOFA scores of $>6$. The scores significantly differed between the two patient groups $(p<0.05)$. The serum expression of miR-7110-5p did not significantly differ for patients with various SOFA scores.

Among the 44 enrolled patients with sepsis secondary to pneumonia, 23 survived (52.3\%), whereas 21 died (47.7\%). Table 4 shows the clinical characteristics of patients with sepsis. The plasma levels of miR-7110-5p and miR-223-3p did not significantly differ between the patients who survived and died of sepsis (Table 5).

\section{Discussion}

miRNAs have been used as biomarkers for various cancer types since the discovery of circulating miRNAs in the human peripheral sera. Several circulating miRNAs have been identified as potential biomarkers for sepsis, but their roles in infectious diseases were rarely investigated [1117]. The association of miRNAs with the diagnosis of sepsis has been studied but remains controversial. Sepsis has various causes, such as severe wound, burn, shock, infection, and surgical operation; such causes present similarities and differences. No research has investigated the role of miRNAs in sepsis secondary to pneumonia and their relation to the diagnosis of sepsis.

In this study, the expression levels of miR-7110-5p and miR-223-3p differed among healthy controls, patients with pneumonia, and patients with sepsis secondary to pneumonia. The expression levels of these miRNAs were upregulated in the plasma of patients with sepsis secondary to pneumonia. The AUCs of the ROC of miR-7110-5p and miR-223-3p for the prediction of 

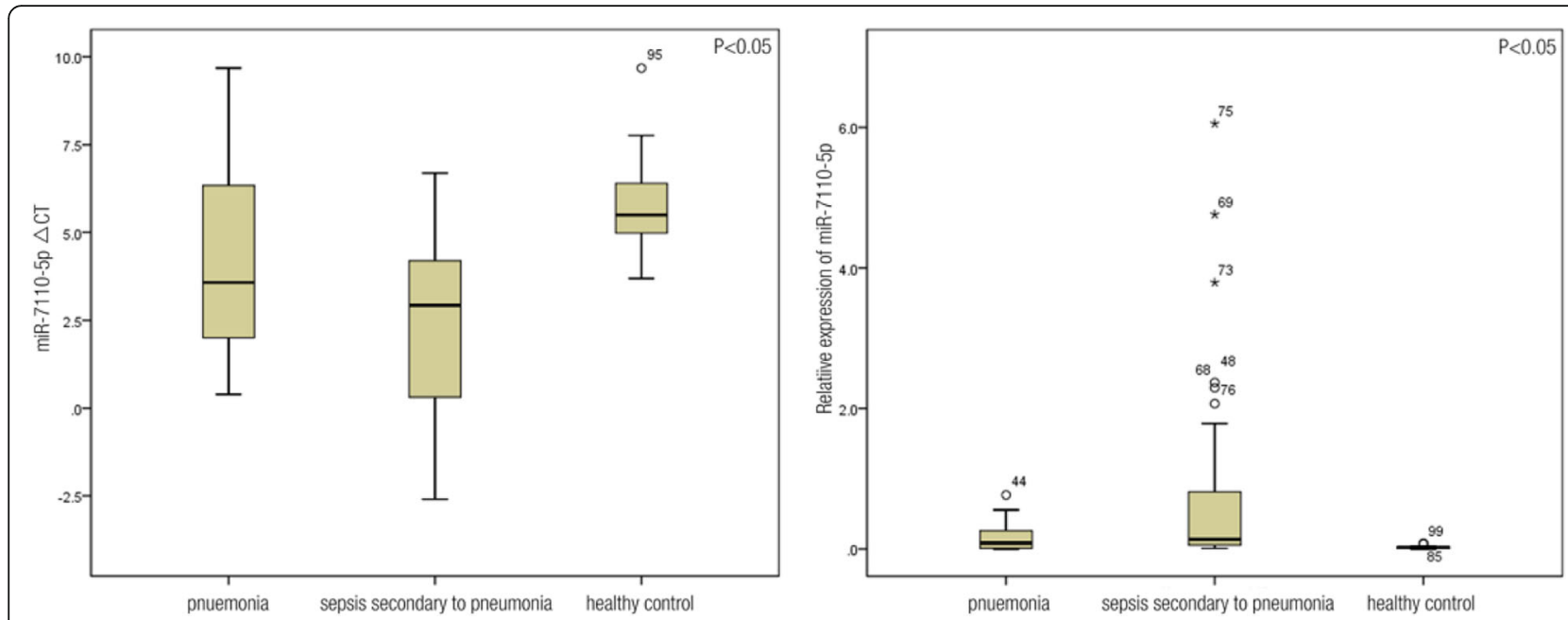

Fig. 2 Image on the left shows that the $\Delta C T$ values of miR-7110-5p in the pneumonia, sepsis, and healthy control groups were $4.15 \pm 2.52,2.27 \pm 2.64$, and $5.73 \pm 1.35$, respectively, which were significantly different $(p=0.000)$. Moreover, the $\Delta C T$ of miR-7110-5p significantly differed between the healthy control and pneumonia groups $(p=0.002)$, healthy control and sepsis groups $(p=0.000)$, and pneumonia and sepsis groups $(p=0.001)$. Image on the right shows that the relative expression levels of miR-7110-5p in the three groups were $0.084(0.012,0.28), 0.133(0.054,0.963)$, and $0.022(0.011,0.033)$, which were significantly different $(p=0.000)$. Moreover, the relative expression of miR-7110-5p significantly differed between healthy control and pneumonia groups $(p=0.015)$, healthy control and sepsis groups $(p=0.000)$, and pneumonia group and sepsis groups $(p=0.006)$

sepsis secondary to pneumonia were 0.883 and 0.964 , respectively. This finding indicated the potential use of these miRNAs as biomarkers for sepsis.

In 2009, Vasilescu [11] first reported that miR-150 in the plasma can be used as a serum biomarker to diagnose patients with sepsis. The expression profiles of miR-150, miR-182, miR-342-5p, and miR-486 were used to distinguish patients with sepsis from healthy controls through genome-wide miRNA profiling by using microarray analysis of the peripheral blood leukocytes of eight healthy individuals and eight patients with sepsis. This result was confirmed through qRT-PCR analysis. Xie [13] screened sepsis biomarkers in the entire genome and evaluated the relationship of miRNAs to the prognosis of sepsis. The expression levels of miR-223, miR-15a, miR-122, miR-193, and miR483-5p significantly differed between the
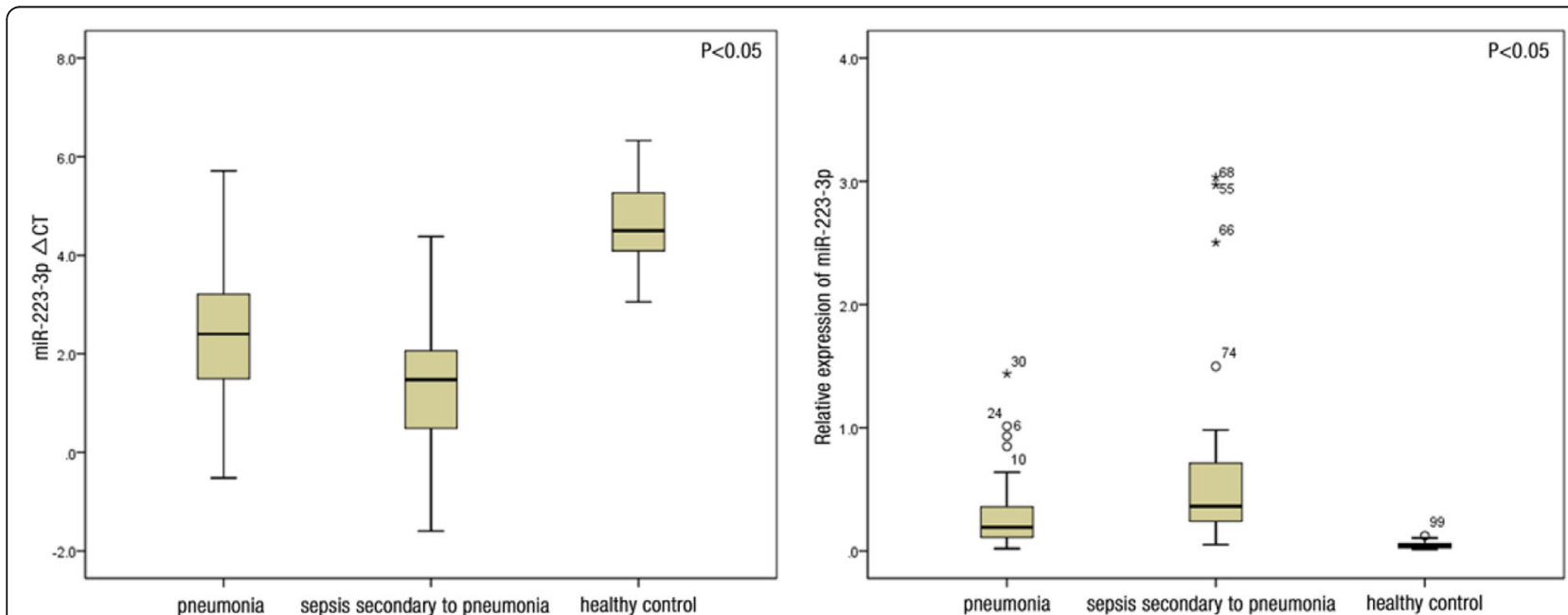

Fig. 3 Image on the left shows that the $\Delta C T$ levels of miR-223-3p in the pneumonia, sepsis, and healthy control groups were $2.39 \pm 1.36,1.44 \pm$ 1.43 , and $4.58 \pm 0.91$, respectively, which were significantly different $(p=0.000)$. The $\Delta C T$ values of miR-223-3p significantly differed between the healthy and pneumonia groups $(p=0.000)$, healthy control and sepsis groups $(p=0.000)$, pneumonia and sepsis groups $(p=0.002)$. Image on the right shows that the relative expression levels of miR-223-3p in the three groups were $0.189(0.107,0.367), 0.361(0.221,0.735)$, and $0.044(0.022$, 0.061 ), which were significantly different $(p=0.000)$. In addition, the relative expression level of miR-223-3p significantly differed between the healthy control and pneumonia groups $(p=0.000)$, healthy control and sepsis groups $(p=0.000)$, and pneumonia and sepsis groups $(p=0.004)$ 

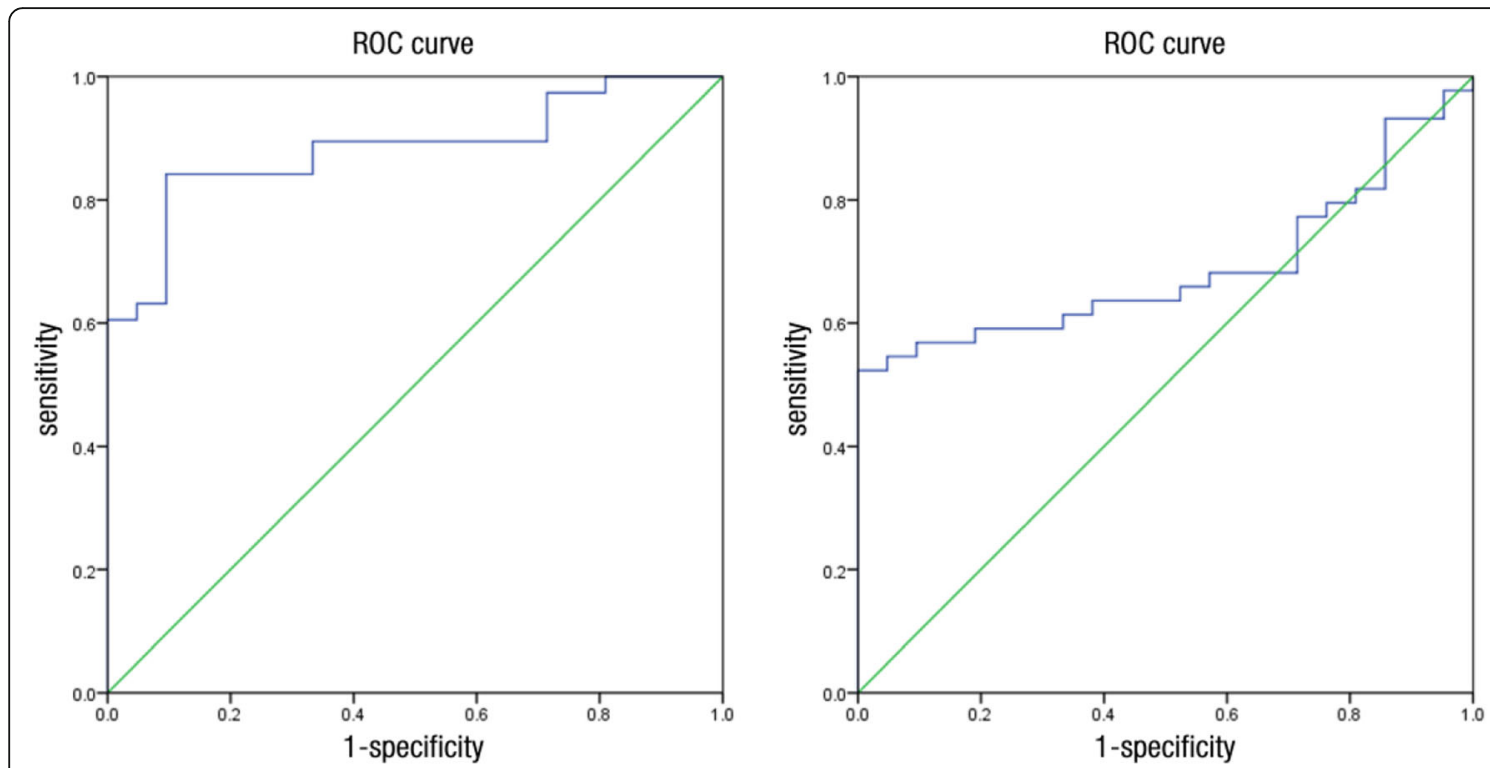

reference line

miR-7110-5p

Fig. 4 ROC curve of miR-7110-5p. Image on the left shows that the AUC of the ROC of miR-7110-5p for the prediction of pneumonia was 0.687. Image on the right shows that the AUC of the ROC of miR-7110-5p for sepsis prediction secondary to pneumonia was 0.883

surviving and deceased group of patients with sepsis. The combination of six miRNAs with acute physiology and chronic health score (Acute Physiology Chronic Health Evaluation II) predicts sepsis and provides a sensitivity of $96.9 \%[16]$.

In this study, gene chip analysis revealed that six miRNAs, namely, miR-4800-5p, miR-6510-5p, miR-6740-5p, miR-7110-5p, miR-765, and miR-940, satisfied the screening criteria of $p<0.01$, fold change $\geq 2$ or $<0.5$, and copy number $>50$. Limited data have been presented regarding the relationship of these six miRNAs to sepsis because of the following factors: 1) sepsis displays various causes, and sepsis secondary to pneumonia has yet to be investigated in terms of the association of miRNAs with the diagnosis of sepsis; 2) unlike in previous studies, we employed Sepsis 3.0 to establish the diagnosis of sepsis; 3 ) the number of samples in the gene chip analysis was limited. Four miRNAs associated with sepsis in previous reports were verified through qRT-PCR analysis to compensate for the limitations of our study.
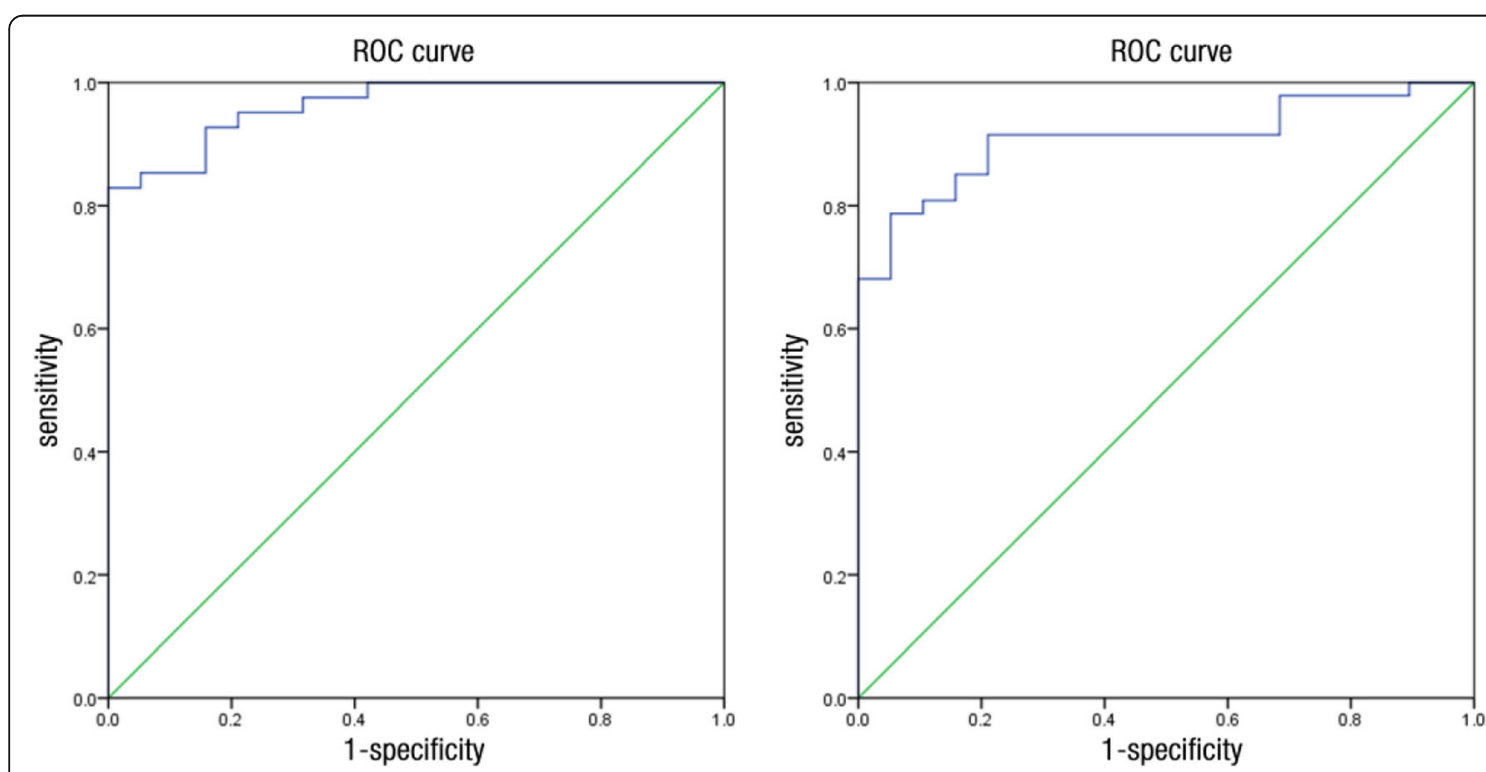

reference line

miR-223-3p

Fig. 5 ROC curve of miR-223-3p. Image on the left shows that the AUC of the ROC of miR-223-3p for the prediction of pneumonia was 0.909 . Image on the right shows that the AUC of the ROC of miR-223-3p for sepsis prediction secondary to pneumonia was 0.964 
Table 4 Clinical characteristic of 44 cases of sepsis secondary to pneumonia

\begin{tabular}{|c|c|c|c|c|}
\hline & & Survivor $(n)$ & Dead $(n)$ & $P$ value \\
\hline \multirow[t]{2}{*}{ Sex } & Male & 21 & 13 & \\
\hline & Female & 2 & 8 & \\
\hline Age & & $57.30 \pm 21.51$ & $70.76 \pm 15.40$ & 0.023 \\
\hline SOFA score & & $5(4,7)$ & $7(4,9.5)$ & 0.010 \\
\hline SOFA above baseline & & $5(3,6)$ & $7(4,9)$ & 0.016 \\
\hline SOFA score (oxygenation index) & & $2(2,3)$ & $3(2.5,4)$ & 0.024 \\
\hline \multirow[t]{2}{*}{ coagulation disorder } & No & $14(60.9 \%)$ & $10(47.6 \%)$ & 0.378 \\
\hline & Yes & $9(39.1 \%)$ & $11(52.4 \%)$ & \\
\hline \multirow[t]{2}{*}{ liver dysfunction } & No & $17(73.9 \%)$ & 19 (90.5\%) & 0.245 \\
\hline & Yes & $6(26.1 \%)$ & $2(9.5 \%)$ & \\
\hline \multirow[t]{2}{*}{ circulatory dysfunction } & No & $18(78.3 \%)$ & $13(61.9 \%)$ & 0.325 \\
\hline & Yes & $5(21.7 \%)$ & $8(47.7 \%)$ & \\
\hline \multirow[t]{2}{*}{ disturbance of consciousness } & No & $15(65.2 \%)$ & $8(38.1 \%)$ & 0.070 \\
\hline & Yes & $8(34.8 \%)$ & $13(61.9 \%)$ & \\
\hline \multirow[t]{2}{*}{ renal dysfunction } & No & $17(73.9 \%)$ & $12(57.1 \%)$ & 0.240 \\
\hline & Yes & $6(26.1 \%)$ & $9(42.9 \%)$ & \\
\hline $\mathrm{PCT}(\mathrm{ng} / \mathrm{mL})$ & & $0.64(0.21,1.86)$ & $0.99(0.51,8.70)$ & 0.109 \\
\hline WBC $\left(10^{9} / \mathrm{L}\right)$ & & $14.4 \pm 10.67$ & $16.01 \pm 10.95$ & 0.624 \\
\hline CRP (mg/dL) & & $87.35 \pm 55.79$ & $116.08 \pm 69.43$ & 0.141 \\
\hline \multirow[t]{2}{*}{ Merges underlying disease } & No & 3 & 10 & 0.012 \\
\hline & Yes & 20 & 11 & \\
\hline Hospitalization days in ICU & & $17.13(10.00,22.00)$ & $11.09(3.00,14.00)$ & 0.003 \\
\hline
\end{tabular}

In this study, miR-223-3p was highly expressed in the circulating blood of patients with sepsis secondary to pneumonia. Wang et al. [12] compared the levels of miR-223 among 50 patients with sepsis, patients with systemic inflammatory response syndrome (SIRS), and healthy controls. The miR-223 expression level increased in the blood of patients with sepsis and SIRS caused by infection but not in the blood of patients with non-infectious SIRS. Hence, miR-223 can be used as a biomarker to distinguish infectious SIRS from non-infectious SIRS. A cohort study involving children with sepsis and healthy ones revealed that the expression levels of miR-223 and miR-146a substantially increased; the increase in miR-223 level was positively associated with a high level of tumor necrosis factor- $\alpha$, disease severity, and poor prognosis [18]. However, these conclusions are inconsistent with other published reports. Clinical studies on non-infectious critically ill patients and patients with sepsis indicated that miR-223 levels with no

Table $5 \Delta C T$ of miR-223-3p and miR-7110-5p between the sepsis death and survival

\begin{tabular}{llll}
\hline$\Delta C T$ & Survival $(n=23)$ & Death $(n=21)$ & $P$ Value \\
\hline miR-223-3p & $1.72 \pm 1.07$ & $0.89 \pm 0.24$ & 0.056 \\
miR-7110-5p & $2.51 \pm 1.02$ & $2.01 \pm 0.87$ & 0.598 \\
\hline
\end{tabular}

significantly difference [19] . Our previous studies on the efficacy of miR-223-3p in predicting sepsis secondary to pneumonia revealed that the AUC of the ROC curve was 0.964, thereby suggesting that the miR-223-3p expression level can be an accurate predictor for the diagnosis of sepsis. In the present study, the expression of miR-7110-5p, which has been rarely examined, was upregulated in the circulating blood of patients with sepsis secondary to pneumonia. However, the signal of miR-7110-5p was altered not only because of the presence of pneumonia or sepsis but also possibly because of the conditions induced by pneumonia or sepsis. Fourteen miRNAs differed in terms of their expression levels in cancer stem cells, CD133(+) A549 cells, and CD133(-) cells. Among these miRNAs, five were upregulated (hsa-miR-23b-3p, -23a-3p, -15b-5p, - 24-3p, and-4734), whereas nine were downregulated (hsa-miR-1246, -30b-5p, - 5096, - 6510-5p, has-miR-7110-5p, $-7641,-3197,-7108-5 p$, and - 6791-5p). Although miR-7110-5p was downregulated [20], its relationship to sepsis has not been studied.

This research presented several limitations. In the RT-PCR validation of miRNAs, we enrolled 52 patients with pneumonia, 44 patients with pneumonia who satisfied the Sepsis 3.0 diagnostic criteria, and 21 healthy controls. The total number of cases enrolled in the study was limited. 
Hence, the value of miR-7110-5p and miR-223-3p in predicting early sepsis secondary to pneumonia should be further evaluated using large samples.

\section{Conclusion}

Agilent miRNA chip technology was applied to identify differentially expressed miRNAs in the plasma of patients with pneumonia and sepsis secondary to pneumonia (Sepsis 3.0). qRT-PCR results revealed that miR-7110-5p and miR-223-3p were upregulated in the plasma of patients with sepsis secondary to pneumonia. This study identified miR-223-3p as a promising biomarker for sepsis due to pneumonia. These results could serve as basis for future study design with an adequate sample size to verify the clinical usefulness of miRNAs as respiratory sepsis biomarkers.

\section{Abbreviations \\ AUC: Area under the curve; CAP: Community-acquired pneumonia; CV: Coefficient of Variance; HAP: Hospital-Acquired Pneumonia; miRNA: MicroRNA; MODS: Multiple Organ Dysfunction Syndrome; qRT- PCR: Quantitative Reverse-Transcription Polymerase Chain Reaction; ROC: Receiver operating characteristic curve; SIRS: Systemic Inflammatory Response Syndrome; SOFA: Sequential Organ Failure Assessment}

\section{Acknowledgments}

We gratefully acknowledge the participation of all critical care physicians and nurses in the Department of Respiratory and Critical Care Medicine of the People's Hospital of Zhengzhou University.

\section{Funding}

This work was supported by the Basic and Frontier Research Program of Science and Technology Department Foundation of Henan Province (Grant No. 152300410148). Funds are used for the design of the study and collection, analysis, and interpretation of data and writing the manuscript.

\section{Availability of data and materials}

The datasets used and/or analyzed are available from corresponding author upon reasonable request.

\section{Authors' contributions \\ WZ substantially contributed to the conception, design, data analysis, data interpretation, drafting, and critical revision of the manuscript. GZ remarkably contributed to the conception and design of the work. JJ participated in the experiment, data collection, and revision of the manuscript. ZL and DS developed the new software used in the work and prepared the draft. LM interpreted the data. All authors have read and approved the final version of the manuscript and agreed to be personally accountable for their contributions and questions related to the accuracy or integrity of any part of the work.}

\section{Ethics approval and consent to participate}

The design of the study was supported by the Basic and Frontier Research Program of the Science and Technology Department Foundation of Henan Province (Grant No. 152300410148). All subjects were approached for written informed consent to participate in the study.

\section{Consent for publication}

Not applicable.

\section{Competing interests}

The authors declare that they have no competing interests.

\section{Publisher's Note}

Springer Nature remains neutral with regard to jurisdictional claims in published maps and institutional affiliations.
Received: 16 April 2018 Accepted: 24 March 2019

Published online: 14 May 2019

\section{References}

1. Danai P, Martin GS. Epidemiology of sepsis: recent advances [J]. Curr Infect Dis Rep. 2005:7(5):329-34.

2. Vincent JL. Management of sepsis in the critically ill patient: key aspects [J]. Expert Opin Pharmacother. 2006;7(15):2037-45.

3. Angus DC, Van Der Poll T. Severe sepsis and septic shock [J]. N Engl J Med. 2013;369(21):2063.

4. Levy MM, Artigas A, Phillips GS, Rhodes A, Beale R, Osborn T, Vincent JL, Townsend S, Lemeshow S, Dellinger R. Outcomes of the surviving Sepsis campaign in intensive care units in the USA and Europe: a prospective cohort study [J]. Lancet Infect Dis. 2012;12(12):919-24.

5. Dellinger RP, Levy MM, Rhodes A, Annane D, Gerlach H, Opal SM, Sevransky JE, Sprung CL, Douglas IS, Jaeschke R, et al. Surviving Sepsis campaign: international guidelines for management of severe sepsis and septic shock, 2012 [J]. Intensive Care Med. 2013;39(2):165-228

6. Lee RC, Feinbaum RL, Ambros V. The C. Elegans heterochronic gene lin-4 encodes small RNAs with antisense complementarity to lin-14 [J]. Cell. 1993: 75(5):843-54.

7. Essandoh K, Fan GC. Role of extracellular and intracellular microRNAs in sepsis [J]. Biochim Biophys Acta. 2014;1842(11):2155-62.

8. Kalil AC, Metersky ML, Klompas M, Muscedere J, Sweeney DA, Palmer LB, Napolitano LM, O'grady NP, Bartlett JG, Carratalà J. Executive summary: Management of Adults with Hospital-acquired and Ventilator-associated Pneumonia: 2016 clinical practice guidelines by the Infectious Diseases Society of America and the American Thoracic Society []]. Clin Infect Dis. 2016;63(5):575-82.

9. Mandell LA, Whitney CG. Infectious Diseases Society of America/American Thoracic Society consensus guidelines on the Management of CommunityAcquired Pneumonia in adults [J]. Clinical Infectious Diseases An Official Publication of the Infectious Diseases Society of America. 2007;44(Suppl 2 (Supplement 2):S27-72.

10. Singer M, Deutschman C S, Seymour C W, Shankar-Hari M, Annane D, Bauer M, Bellomo R, Bernard G R, Chiche J D, Coopersmith C M. The third international consensus definitions for Sepsis and septic shock (Sepsis-3) [J]. Jama, 2016, 315 (8): 775

11. Vasilescu C, Rossi S, Shimizu M, Tudor S, Veronese A, Ferracin M, Nicoloso MS, Barbarotto E, Popa M, Stanciulea O. MicroRNA fingerprints identify miR150 as a plasma prognostic marker in patients with sepsis [J]. PLoS One. 2009:4(10):e7405.

12. Wang JF, Yu ML, Yu G, Bian JJ, Deng XM, Wan XJ, Zhu KM. Serum miR-146a and miR-223 as potential new biomarkers for sepsis [J]. Biochem Biophys Res Commun. 2010;394(1):184-8.

13. Wang $H$, Zhang $P$, Chen W, Feng D, Jia Y, Xie L. Serum microRNA signatures identified by Solexa sequencing predict sepsis patients' mortality: a prospective observational study [J]. PLoS One. 2012;7(6):e38885.

14. Wang HJ, Zhang PJ, Chen WJ, Feng D, Jia YH, Xie LX. Four serum microRNAs identified as diagnostic biomarkers of sepsis [J]. J Trauma Acute Care Surg. 2012:73(4):850-4.

15. Wang HJ, Deng J, Wang JY, Zhang PJ, Xin Z, Xiao K, Feng D, Jia YH, Liu YN, Xie LX. Serum miR-122 levels are related to coagulation disorders in sepsis patients [J]. Clinical chemistry. Lab Med. 2014;52(6):1-7.

16. Wang HJ, Zhang PJ, Chen WJ, Jie D, Dan F, Jia YH, Xie LX. Characterization and identification of novel serum microRNAs in sepsis patients with different outcomes []]. Shock. 2013;39(6):480.

17. Ledderose C, Möhnle P, Limbeck E, Schütz S, Weis F, Rink J, Briegel J, Kreth S. Corticosteroid resistance in sepsis is influenced by microRNA-124-induced downregulation of glucocorticoid receptor-a []]. Crit Care. 2012;16(3):2745-53.

18. Wu Y, Li C, He Y, Li Q, Wang G, Wen P, Yang W, Yang Y. [Relationship between expression of microRNA and inflammatory cytokines plasma level in pediatric patients with sepsis] [J]. Zhonghua Er Ke Za Zhi Chinese J Pediatr, 2014, 52 (1): 28-33.

19. Benz F, Tacke F, Luedde M, Trautwein C, Luedde T, Koch A, Roderburg C. Circulating MicroRNA-223 serum levels do not predict Sepsis or survival in patients with critical illness [J]. Dis Markers. 2015;2015:384208.

20. Chen QY, Jiao DM, Zhu Y, Hu H, Wang J, Tang X, Chen J, Yan L. Identification of carcinogenic potential-associated molecular mechanisms in CD133(+) A549 cells based on microRNA profiles []]. Tumor Biol. 2016;37(1):521-30. 